\title{
Kinematics and Dynamics of an Asymmetrical Parallel Robotic Wrist
}

\author{
Guanglei Wu \\ Department of Mechanical and Manufacturing Engineering, Aalborg University, 9220 Aalborg, Denmark
}

Correspondence should be addressed to Guanglei Wu; gwu@m-tech.aau.dk

Received 24 July 2014; Accepted 1 November 2014; Published 1 December 2014

Academic Editor: Gordon R. Pennock

Copyright (C) 2014 Guanglei Wu. This is an open access article distributed under the Creative Commons Attribution License, which permits unrestricted use, distribution, and reproduction in any medium, provided the original work is properly cited.

This paper introduces an asymmetrical parallel robotic wrist, which can generate a decoupled unlimited-torsion motion and achieve high positioning accuracy. The kinematics, dexterity, and singularities of the manipulator are investigated to visualize the performance contours of the manipulator. Using the method of Lagrange multipliers and considering all the mobile components, the equations of motion of the manipulator are derived to investigate the dynamic characteristics efficiently. The developed dynamic model is numerically illustrated and compared with its simplified formulation to show its computation accuracy.

\section{Introduction}

The parallel typed wrist mechanisms, also called spherical parallel manipulators (SPMs), have found their applications in camera-orientating [1], minimally invasive surgery [2] and wrist joint [3] thanks to their large orientation workspace and high payload capacity. Since the SPM can generate three pure rotations, another potential application is that it can function as a tool head for complicated surface machining. However, the general SPM only can produce a limited torsion motion with a prescribed tilt angle, whereas an unlimited torsion is necessary in some common material processing such as milling. The coaxial input SPM reported in [3] can achieve unlimited torsion, whereas its unique structure introduces a complex input mechanism. Moreover, the general SPMs result in low positioning accuracy [4] without a ball-and-socket joint as the center of rotation. In this paper, an asymmetrical parallel robotic wrist is proposed, which can generate an unlimited-torsion motion with enhanced positioning accuracy. This manipulator adopts a universal joint as the center of rotation supported by an input shaft at the center, which simplifies the manipulator architecture.

The SPMs have been extensively studied on many aspects, such as workspace [5, 6], dexterity [7-9], singularity [10], stiffness [4,11], and type synthesis [12-14]. These performance criteria can be classified into two groups: one relates to the kinematic performance of the manipulator while the other relates to the kinetostatic/dynamic performance of the manipulator [15]. In the kinematic considerations, the quality of the workspace that reflects the shape, size, and presence of singularities is of primary importance in the manipulator design. Another utmost important concern is the dexterity, which is usually evaluated by means of the conditioning number of the kinematic Jacobian matrix. On the other hand, the dynamics of SPMs received relatively less attention. When the wrist mechanisms are used to develop active spherical joints, the dynamic characteristics are of importance in their design and applications. The most used approaches to derive the equations of motion include recursive Newton-Euler approach and the Lagrange equations [16-18], which have been applied to different parallel robotics. The recursive Newton-Euler method consists of two steps: the first step is an outward recursion to calculate velocity and acceleration from the base to the end link; the second step is an inward recursion to calculate the forces and torques at joints from the end link to the base. By virtue of the principle of virtual work, Staicu [19] used this method to derive the inverse dynamics of the Agile Wrist [11]. The equations of motion for a manipulator can also be formulated with Lagrangian method, which includes two types: one with dependent coordinates and the other with independent coordinates [20]. This paper adopts the first type to develop a dynamic model with the approach of Lagrange multipliers. 


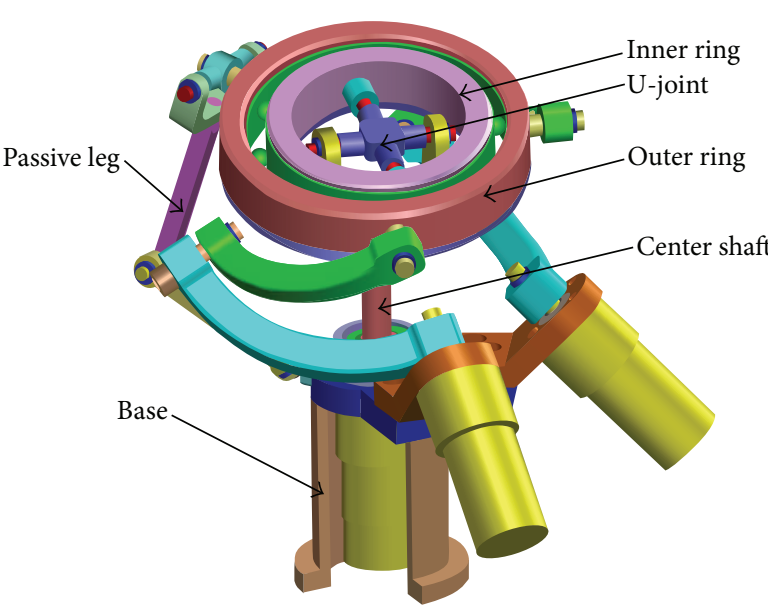

(a)

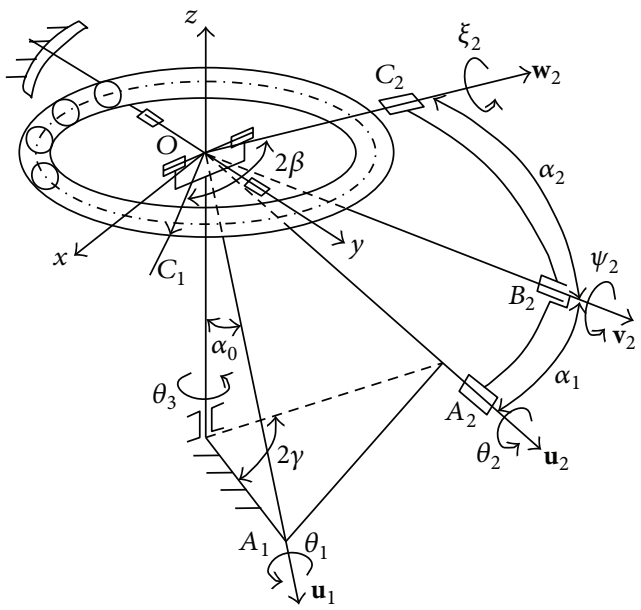

(b)

FIgURE 1: The asymmetrical spherical parallel manipulator: (a) CAD model; (b) coordinate system.

This paper introduces an asymmetrical parallel robotic wrist and presents the kinematic/dynamic analysis. The isocontours of the dexterity over workspace are visualized to show an image of the overall performance and singularity is analyzed. Using the method of Lagrange multipliers and considering all the mobile components, the equations of motion of the manipulator are derived to compute the power consumption efficiently. The developed dynamic model is numerically illustrated and compared with its simplified model.

\section{Manipulator under Study}

The proposed asymmetrical wrist mechanism is displayed in Figure 1(a), whose mobile platform (MP) is composed of an outer and inner ringer via bearing. The orientation of the outer ring is determined by two RRR (throughout this paper, $\mathrm{R}, \mathrm{U}$, and $\mathrm{P}$ stand for revolute, universal, and prismatic joints, resp., and an underlined letter indicates an actuated joint) legs and constrained in a vertical plane by a fully passive RRU leg or an alternative RPU one. Through a U-joint, the decoupled rotation of the inner ringer is driven by the center shaft that supports the MP to improve the positioning accuracy. This manipulator can generate an unlimited torsion, which can be used in milling and work as an active spherical joint.

The coordinate system $(x, y, z)$ for the manipulator is built as shown in Figure 1(b), of which the origin is located at the center of rotation, namely, point $O$. The axes of the three revolute joints in the $i$ th $\underline{R} R R$ leg are parallel to unit vectors $\mathbf{u}_{i}$, $\mathbf{v}_{i}$, and $\mathbf{w}_{i}$, respectively. Both of these two legs have identical proximal and distal curved links defined by $\alpha_{1}$ and $\alpha_{2}$. The base platform is defined by $\gamma$ and $\alpha_{0}$, and the connection joint on the mobile platform is defined by $\beta$.

\section{Kinematics of the Manipulator}

The orientation of the outer ring of the mobile platform is presented by Euler angles $\left(\phi_{x}, \phi_{y}\right)$, for which the rotation matrix is expressed as $\mathbf{Q}=\mathbf{R}\left(\mathbf{x}, \phi_{x}\right) \mathbf{R}\left(\mathbf{y}, \phi_{y}\right)$. Hence, the orientation of the inner ring can be described with Cardan angles $\left(\phi_{x}, \phi_{y}, \phi_{z}\right)$ which yields the rotation matrix $\mathbf{R}=$ $\mathbf{Q R}\left(\mathbf{z}, \phi_{z}\right)$, and its output axis is denoted by $\mathbf{p}=\mathbf{Q z}$, where $\mathbf{z}=[0,0,1]^{T}$.

Under the prescribed coordinate system, unit vector $\mathbf{u}_{i}$ is derived as

$\mathbf{u}_{i}=\left[\begin{array}{lll}(-1)^{i+1} \sin \gamma \sin \alpha_{0} & \cos \gamma \sin \alpha_{0}-\cos \alpha_{0}\end{array}\right]^{T}, \quad i=1,2$.

Unit vector $\mathbf{v}_{i}$ of the axis of the intermediate revolute joint in the $i$ th leg is expressed as

$$
\begin{aligned}
\mathbf{v}_{i} & =\left[\begin{array}{l}
a_{i} \\
b_{i} \\
c_{i}
\end{array}\right] \\
& =\left[\begin{array}{c}
(-1)^{i+1} s \gamma s \alpha_{0} c \alpha_{1}+\left(s \theta_{1} c \gamma+(-1)^{i+1} c \theta_{1} s \gamma c \alpha_{0}\right) s \alpha_{1} \\
c \gamma s \alpha_{0} c \alpha_{1}+\left((-1)^{i} s \theta_{1} s \gamma+c \theta_{1} c \gamma c \alpha_{0}\right) s \alpha_{1} \\
-c \alpha_{0} c \alpha_{1}+c \theta_{1} s \alpha_{0} s \alpha_{1}
\end{array}\right], \\
& i=1,2,
\end{aligned}
$$

where $s$ and $\mathrm{c}$ stand for the sine and cosine functions, respectively.

Unit vector $\mathbf{w}_{i}$ of the top revolute joint in the $i$ th leg is a function of the outer ring orientation:

$$
\begin{gathered}
\mathbf{w}_{i}=\left[\begin{array}{lll}
x_{i} & y_{i} & z_{i}
\end{array}\right]^{T}=\mathbf{Q} \mathbf{w}_{i}^{*} ; \\
\mathbf{w}_{i}^{*}=\left[\begin{array}{lll}
(-1)^{i+1} \sin \beta & \cos \beta & 0
\end{array}\right]^{T},
\end{gathered}
$$

where $\mathbf{w}_{i}^{*}$ is the unit vector for the axis of the top revolute joint of the $i$ th leg when the mobile platform is at its home configuration. 
3.1. Inverse Geometry. Following the kinematic constraint equations below,

$$
\mathbf{v}_{i} \cdot \mathbf{w}_{i}-\cos \alpha_{2}=0, \quad i=1,2 \text {. }
$$

The spherical trigonometry equation for the $i$ th $\underline{R R R}$ legs is written as

$$
A_{i} t_{i}^{2}+2 B_{i} t_{i}+C_{i}=0, \quad i=1,2
$$

with

$$
\begin{aligned}
A_{i}= & \left((-1)^{i+1} x_{i} \mathrm{~s} \gamma+y_{i} \mathrm{c} \gamma\right) \mathrm{s}\left(\alpha_{0}-\alpha_{1}\right) \\
& -z_{i} \mathrm{c}\left(\alpha_{0}-\alpha_{1}\right)-\mathrm{c} \alpha_{2}, \\
B_{i}= & \left(x_{i} \mathrm{c} \gamma-y_{i} \mathrm{~s} \gamma\right) \mathrm{s} \alpha_{1}, \\
C_{i}= & \left((-1)^{i+1} x_{i} \mathrm{~s} \gamma+y_{i} \mathrm{c} \gamma\right) \mathrm{s}\left(\alpha_{0}+\alpha_{1}\right) \\
& -z_{i} \mathrm{c}\left(\alpha_{0}+\alpha_{1}\right)-\mathrm{c} \alpha_{2} .
\end{aligned}
$$

The input angle displacements can be solved as

$$
\begin{gathered}
\cos \theta_{i}=\frac{1-t_{i}^{2}}{1+t_{i}^{2}}, \quad \sin \theta_{i}=\frac{2 t_{i}}{1+t_{i}^{2}} \\
t_{i}=\tan \frac{\theta_{i}}{2}=\frac{-B_{i} \pm \sqrt{B_{i}^{2}-A_{i} C_{i}}}{A_{i}} .
\end{gathered}
$$

Solving (5), the inverse geometry problem has four solutions corresponding to the four working modes, which is characterized by the sign $\langle-/+\rangle$ of $\mathbf{u}_{i} \times \mathbf{v}_{i} \cdot \mathbf{w}_{i}$. Here, the $\langle-+\rangle$ mode is selected as the working mode.

According to the motion of U-joint [21], the input angle $\theta_{3}$ of the center shaft is derived as follows:

$$
\theta_{3}=\tan ^{-1}\left(\tan \phi_{z} \cos \phi_{x} \cos \phi_{y}\right)
$$

3.2. Kinematic Jacobian Matrix. Differentiating (4) and (8) with respect to time, the relationship between the angle rates and the input velocity is derived as

$$
\left[\begin{array}{ccc}
A_{11} & A_{12} & 0 \\
A_{21} & A_{22} & 0 \\
A_{31} & A_{32} & A_{33}
\end{array}\right]\left[\begin{array}{l}
\dot{\phi}_{x} \\
\dot{\phi}_{y} \\
\dot{\phi}_{z}
\end{array}\right]=\left[\begin{array}{ccc}
B_{11} & 0 & 0 \\
0 & B_{22} & 0 \\
0 & 0 & -1
\end{array}\right]\left[\begin{array}{c}
\dot{\theta}_{1} \\
\dot{\theta}_{2} \\
\dot{\theta}_{3}
\end{array}\right]
$$

or $\mathbf{A} \dot{\phi}=\mathbf{B} \dot{\theta}$ with

$$
\begin{aligned}
& A_{i 1}=b_{i}\left((-1)^{i+1} \mathrm{c} \phi_{x} s \phi_{y} \mathrm{~s} \beta-\mathrm{s} \phi_{x} \mathrm{c} \beta\right) \\
& +c_{i}\left((-1)^{i} \mathrm{~s} \phi_{x} \mathrm{~s} \phi_{y} \mathrm{~s} \beta+\mathrm{c} \phi_{x} \mathrm{c} \beta\right), \quad i=1,2 \text {, } \\
& A_{i 2}=(-1)^{i} a_{i} s \phi_{y} \mathrm{~s} \beta+(-1)^{i+1} b_{i} s \phi_{x} \mathrm{c} \phi_{y} \mathrm{~s} \beta \\
& +(-1)^{i} c_{i} \mathrm{c} \phi_{x} \mathrm{c} \phi_{y} \mathrm{~s} \beta, \quad i=1,2, \\
& B_{i i}=x_{i} s \alpha_{1}\left(s \theta_{i} s \gamma c \alpha_{0}+(-1)^{i} \mathrm{c} \theta_{i} \mathrm{c} \gamma\right) \\
& +y_{i} s \alpha_{1}\left(\mathrm{~s} \theta_{i} \mathrm{c} \gamma \mathrm{c} \alpha_{0}-(-1)^{i} \mathrm{c} \theta_{i} \mathrm{~s} \gamma\right) \\
& +c_{i} \mathrm{~s} \theta_{i} \mathrm{~s} \alpha_{0} \mathrm{~s} \alpha_{1}, \quad i=1,2, \\
& A_{31}=\frac{\tan \phi_{z} s \phi_{x} c \phi_{y}}{D} \text {, } \\
& A_{32}=\frac{\tan \phi_{z} c \phi_{x} s \phi_{y}}{D} \\
& A_{33}=\frac{-\left(1+\tan ^{2} \phi_{z}\right) c \phi_{x} c \phi_{y}}{D} ; \\
& D=1+\left(\tan \phi_{z} c \phi_{x} c \phi_{y}\right)^{2} \text {. }
\end{aligned}
$$

Since the angle rates can be transformed into the angular velocity, namely,

$$
\left[\begin{array}{c}
\omega_{x} \\
\omega_{y} \\
\omega_{z}
\end{array}\right]=\left[\begin{array}{ccc}
\cos \phi_{x} \cos \phi_{z} & \sin \phi_{z} & 0 \\
-\cos \phi_{y} \sin \phi_{z} & \cos \phi_{z} & 0 \\
\sin \phi_{y} & 0 & 1
\end{array}\right]\left[\begin{array}{c}
\dot{\phi}_{x} \\
\dot{\phi}_{y} \\
\dot{\phi}_{z}
\end{array}\right] \quad \text { or } \quad \boldsymbol{\omega}=\boldsymbol{\Phi} \dot{\boldsymbol{\phi}}
$$

The kinematic Jacobian matrix of the manipulator is expressed as

$$
\mathbf{J}=\boldsymbol{\Phi} \mathbf{A}^{-1} \mathbf{B}
$$

Equation (9) or $\boldsymbol{\omega}=\mathbf{J} \dot{\boldsymbol{\theta}}$ relates the motions of the inner ring of the mobile platform to the input angular velocities.

3.3. Motions of the Manipulator. This section presents the velocity equations for the mobile bodies of the manipulator and the acceleration equations can be derived by differentiating the velocity equations with respect to time.

3.3.1. Outer Ring of MP. Due to the decoupled motion of the outer ring of the mobile platform, its angle rates can be extracted from (9) as follows:

$$
\left[\begin{array}{ll}
A_{11} & A_{12} \\
A_{21} & A_{22}
\end{array}\right]\left[\begin{array}{l}
\dot{\phi}_{x} \\
\dot{\phi}_{y}
\end{array}\right]=\left[\begin{array}{cc}
B_{11} & 0 \\
0 & B_{22}
\end{array}\right]\left[\begin{array}{c}
\dot{\theta}_{1} \\
\dot{\theta}_{2}
\end{array}\right] \quad \text { or } \quad \mathbf{A}^{\prime} \dot{\phi}^{\prime}=\mathbf{B}^{\prime} \dot{\boldsymbol{\theta}}^{\prime}
$$




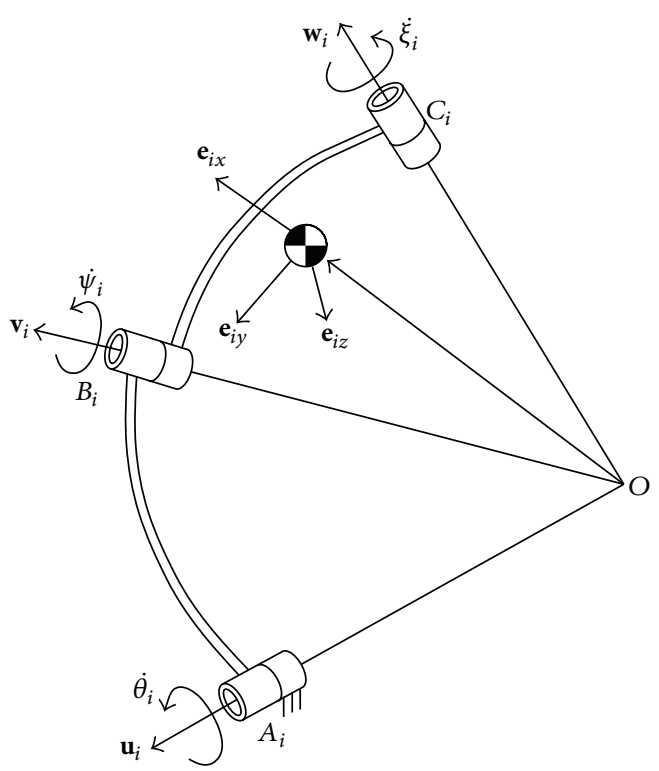

(a)

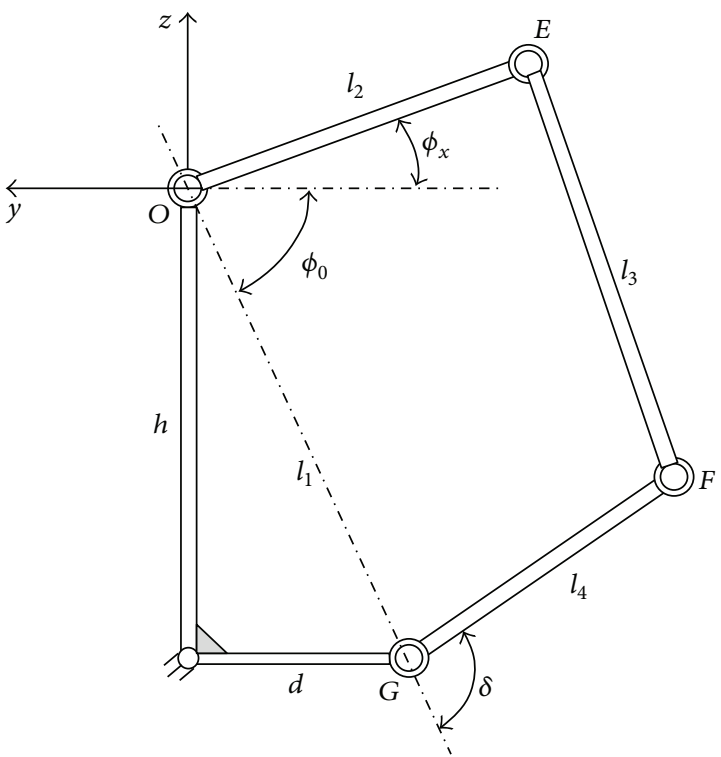

(b)

FIgURE 2: The motions of the legs: (a) active; (b) passive.

On the other hand, the angular velocity $\dot{\boldsymbol{\omega}}^{\prime}$ of the outer ring can be found upon the differentiation of (4) with respect to time, namely,

$$
\begin{aligned}
& {\left[\begin{array}{c}
\left(\mathbf{v}_{1} \times \mathbf{w}_{1}\right)^{T} \\
\left(\mathbf{v}_{2} \times \mathbf{w}_{2}\right)^{T}
\end{array}\right]\left[\begin{array}{c}
\omega_{x}^{\prime} \\
\omega_{y}^{\prime} \\
\omega_{z}^{\prime}
\end{array}\right]} \\
& \quad=\left[\begin{array}{cc}
\left(\mathbf{u}_{1} \times \mathbf{v}_{1}\right) \cdot \mathbf{w}_{1} & 0 \\
0 & \left(\mathbf{u}_{2} \times \mathbf{v}_{2}\right) \cdot \mathbf{w}_{2}
\end{array}\right]\left[\begin{array}{l}
\dot{\theta}_{1} \\
\dot{\theta}_{2}
\end{array}\right] \\
& \text { or } \mathbf{J}_{x} \boldsymbol{w}^{\prime}=\mathbf{J}_{q} \dot{\boldsymbol{\theta}}^{\prime} .
\end{aligned}
$$

In accordance with (11), the following relationship exists:

$$
\left[\begin{array}{c}
\omega_{x}^{\prime} \\
\omega_{y}^{\prime} \\
\omega_{z}^{\prime}
\end{array}\right]=\left[\begin{array}{ccc}
\cos \phi_{x} & 0 & 0 \\
0 & 1 & 0 \\
\sin \phi_{y} & 0 & 0
\end{array}\right]\left[\begin{array}{l}
\dot{\phi}_{x} \\
\dot{\phi}_{y} \\
\dot{\phi}_{z}
\end{array}\right] \quad \text { or } \quad \boldsymbol{\omega}^{\prime}=\boldsymbol{\Phi}^{\prime} \dot{\boldsymbol{\phi}}
$$

3.3.2. Active Leg. Referring to Figure 2(a), the velocity $\dot{\psi}$ of the intermediate joint in $i$ th leg is found by making use of the following equation:

$$
\boldsymbol{\omega}^{\prime}=\dot{\theta}_{i} \mathbf{u}_{i}+\dot{\psi}_{i} \mathbf{v}_{i}+\dot{\xi}_{i} \mathbf{w}_{i}
$$

To eliminate $\dot{\theta}_{i}$ and $\dot{\xi}_{i}$, dot-multiplying equation (16) on both sides with $\mathbf{u}_{i} \times \mathbf{w}_{i}$ yields

$$
\begin{aligned}
& \left(\mathbf{u}_{i} \times \mathbf{w}_{i}\right) \cdot \boldsymbol{\omega}^{\prime}=\dot{\psi}\left(\mathbf{u}_{i} \times \mathbf{w}_{i}\right) \cdot \mathbf{v}_{i} \\
& \text { or } \quad \dot{\psi}=\mathbf{j}_{\psi i}^{T} \boldsymbol{\omega}^{\prime}=\frac{\left(\mathbf{u}_{i} \times \mathbf{w}_{i}\right)^{T}}{\left(\mathbf{u}_{i} \times \mathbf{w}_{i}\right) \cdot \mathbf{v}_{i}} \boldsymbol{\omega}^{\prime} .
\end{aligned}
$$

The angular velocity of the distal link in the $i$ th leg in the reference frame $(x, y, z)$ is found as

$$
\boldsymbol{\omega}_{i}=\dot{\theta}_{i} \mathbf{u}_{i}+\dot{\psi}_{i} \mathbf{v}_{i}
$$

which can be cast in the local frame by the following transformation matrix:

$$
\begin{gathered}
\mathbf{Q}_{i}=\left[\begin{array}{lll}
\mathbf{e}_{i x} & \mathbf{e}_{i y} & \mathbf{e}_{i z}
\end{array}\right] ; \\
\mathbf{e}_{i x}=\frac{\mathbf{v}_{i}+\mathbf{w}_{i}}{\left\|\mathbf{v}_{i}+\mathbf{w}_{i}\right\|}, \quad \mathbf{e}_{i y}=\frac{\mathbf{v}_{i}-\mathbf{w}_{i}}{\left\|\mathbf{w}_{i}-\mathbf{v}_{i}\right\|}, \quad \mathbf{e}_{i z}=\frac{\mathbf{w}_{i} \times \mathbf{v}_{i}}{\left\|\mathbf{w}_{i} \times \mathbf{v}_{i}\right\|} .
\end{gathered}
$$

3.3.3. Passive Leg. As the motion of the passive leg is confined in the vertical plane, the passive leg is considered as a planar four-bar linkage as shown in Figure 2(b), whose input/output (I/O) equation can be written in the nondimensional form [22]:

$$
\begin{aligned}
k_{1} & +k_{2} \cos \delta-k_{3} \cos \left(\phi_{0}+\phi_{x}\right)-\cos \left(\phi_{0}+\phi_{x}\right) \cos \delta \\
& -\sin \left(\phi_{0}+\phi_{x}\right) \sin \delta=0
\end{aligned}
$$

with

$$
\begin{gathered}
k_{1} \equiv \frac{l_{1}^{2}+l_{2}^{2}-l_{3}^{2}+l_{4}^{2}}{2 l_{2} l_{4}}, \\
k_{2} \equiv \frac{l_{1}}{l_{2}}, \quad k_{3} \equiv \frac{l_{1}}{l_{4}} ; \\
l_{1}=\sqrt{h^{2}+d^{2}}, \quad \phi_{0}=\tan ^{-1} \frac{h}{d} .
\end{gathered}
$$

Differentiating (20) with respect to time leads to

$$
K_{1} \dot{\delta}+K_{2} \dot{\phi}_{x}=0
$$


with

$$
\begin{aligned}
K_{1}= & -k_{2} \sin \delta+\cos \left(\phi_{0}+\phi_{x}\right) \sin \delta-\sin \left(\phi_{0}+\phi_{x}\right) \cos \delta \\
K_{2}= & k_{3} \sin \left(\phi_{0}+\phi_{x}\right)+\sin \left(\phi_{0}+\phi_{x}\right) \cos \delta \\
& -\cos \left(\phi_{0}+\phi_{x}\right) \sin \delta
\end{aligned}
$$

After knowing the $\phi_{x}, \dot{\phi}_{x}, \delta$, and $\dot{\delta}$, the linear and angular velocities of the coupler link $v_{l 3}$ and $\omega_{l 3}$ can be found.

\section{Workspace, Dexterity, and Singularity of the SPM}

The workspace (WS) is one of the most important design issues as it defines the working volume of the robot/manipulator and determines the area that can be reached by a reference frame located on the moving platform or endeffector $[23,24]$. The size and shape of the workspace are of primary importance for the global geometric performance evaluations of the manipulators [25]. The workspace size can be defined by its geometric shape parameters like the radius of a cylindrical/spherical workspace or the sides of the cube for a cubic workspace. In the scope of the paper, a spherical cap defined with $\theta \in\left[0,75^{\circ}\right]$ and $\vartheta \in\left(-180^{\circ}, 180^{\circ}\right]$ in Figure 3 is considered as the regular workspace (RWS).

4.1. Dexterity Analysis. Dexterity is another utmost important concern, which is usually evaluated by the condition number of the kinematic Jacobian matrix. The studies on the SPMs show that smaller angles $\alpha_{0}$ can achieve better dexterity $[6,9]$. Figure 4 demonstrates the distribution of the inverse of the condition number of Jacobian matrix based on 2-norm over the workspace, where $\alpha_{0}=30^{\circ}, \alpha_{1}=\alpha_{2}=75^{\circ}$. These performance contours indicate the dexterous WS region for the manipulator with different designs.

To evaluate the kinematic performance over the regular workspace, a commonly used index is the global conditioning index (GCI) [7]. The GCI is defined over the workspace $W$, which is calculated through a discrete approach in practice, namely,

$$
\begin{aligned}
& \text { GCI }=\frac{\int \kappa^{-1}(\mathbf{J}) \mathrm{d} W}{\int \mathrm{d} W} \\
& \text { or } \mathrm{GCI}=\frac{1}{W} \sum_{i=1}^{n} \frac{1}{\kappa_{i}(\mathbf{J})} \Delta W=\frac{1}{n} \sum_{i=1}^{n} \frac{1}{\kappa_{i}(\mathbf{J})},
\end{aligned}
$$

where $n$ is the discrete number. The index obtained through the above equation is an arithmetic mean, which can be replaced with a quadratic mean for a better indication of the dexterity; subsequently, GCI is redefined as

$$
\mathrm{GCI}=\sqrt{\frac{1}{n} \sum_{i=1}^{n} \frac{1}{\kappa_{i}^{2}(\mathrm{~J})}}
$$

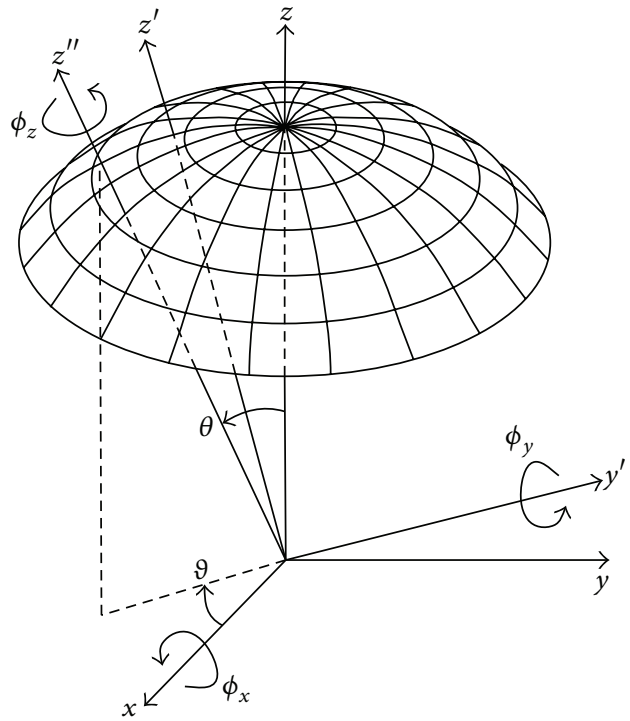

FIGURE 3: The spherical surface of a regular workspace.

TABLE 1: The GCI corresponding to the six designs in Figure 4.

\begin{tabular}{ccccccc}
\hline & (a) & (b) & (c) & (d) & (e) & (f) \\
\hline GCI & 0.230 & 0.305 & 0.258 & 0.310 & 0.139 & 0.280 \\
\hline
\end{tabular}

As a consequence, GCI for the six designs in Figure 4 is computed in Table 1, from which it is seen that the fourth design has the largest value of GCI.

4.2. Singularity Analysis. From Figure 4, it is seen that the smallest value of the isocontours occurs in the workspace center region except Figure 4(e), which implies the possible singularities. Since the input/output of the center shaft is decoupled, which does not have influence on the singular configuration, the singularity is analyzed by means of the forward/backward Jacobians in (14), namely,

$$
\begin{gathered}
\mathbf{J}_{x}=\left[\begin{array}{ll}
\mathbf{v}_{1} \times \mathbf{w}_{1} & \mathbf{v}_{2} \times \mathbf{w}_{2}
\end{array}\right]^{T} ; \\
\mathbf{J}_{q}=\operatorname{diag}\left[\begin{array}{ll}
\left(\mathbf{u}_{1} \times \mathbf{v}_{1}\right) \cdot \mathbf{w}_{1} & \left(\mathbf{u}_{2} \times \mathbf{v}_{2}\right) \cdot \mathbf{w}_{2}
\end{array}\right] .
\end{gathered}
$$

For Type I singularity, it occurs when at least one of the diagonal elements in matrix $\mathbf{J}_{q}$ is equal to zero; that is, the proximal and distal links in a leg are coplanar, leading to

$$
\begin{aligned}
\mathbf{w}_{i} & =\left[\begin{array}{l}
x_{i} \\
y_{i} \\
z_{i}
\end{array}\right] \\
& =\left[\begin{array}{c}
(-1)^{i+1} s \gamma s \alpha_{0} c \alpha_{12}+\left(s \theta_{1} c \gamma+(-1)^{i+1} c \theta_{1} s \gamma c \alpha_{0}\right) s \alpha_{12} \\
c \gamma s \alpha_{0} c \alpha_{12}+\left((-1)^{i} s \theta_{1} s \gamma+c \theta_{1} c \gamma c \alpha_{0}\right) s \alpha_{12} \\
-c \alpha_{0} c \alpha_{12}+c \theta_{1} s \alpha_{0} s \alpha_{12}
\end{array}\right],
\end{aligned}
$$

where $\alpha_{12}=\alpha_{1} \pm \alpha_{2}$. By comparison to (3) and solving the three (overdetermined) equations for each leg, the input 


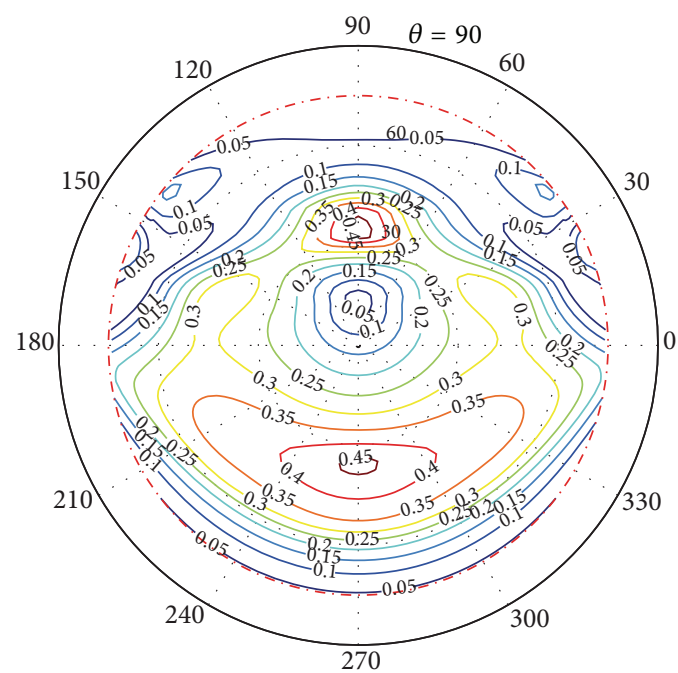

(a) $\gamma=30^{\circ}, \beta=30^{\circ}$

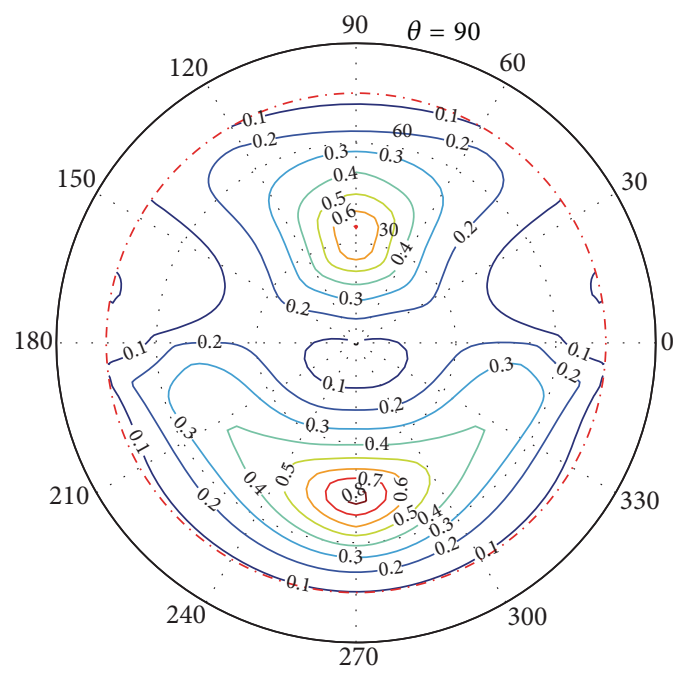

(c) $\gamma=30^{\circ}, \beta=60^{\circ}$

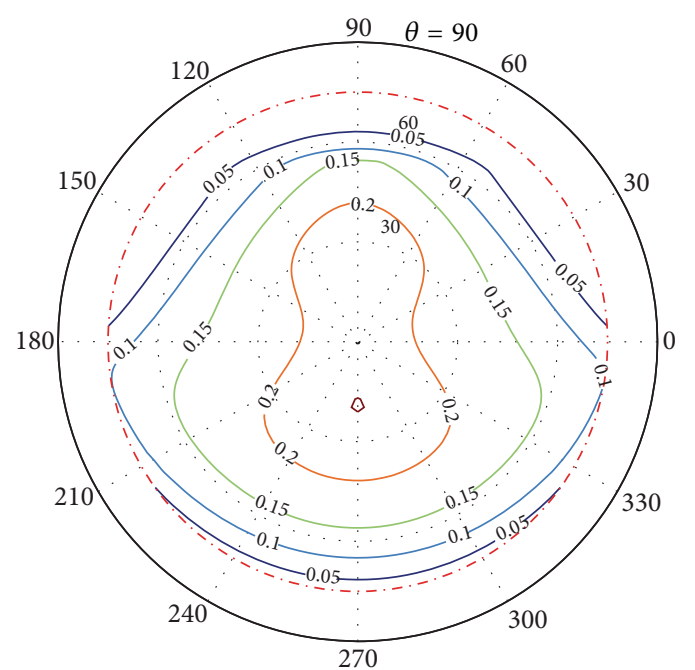

(e) $\gamma=60^{\circ}, \beta=15^{\circ}$

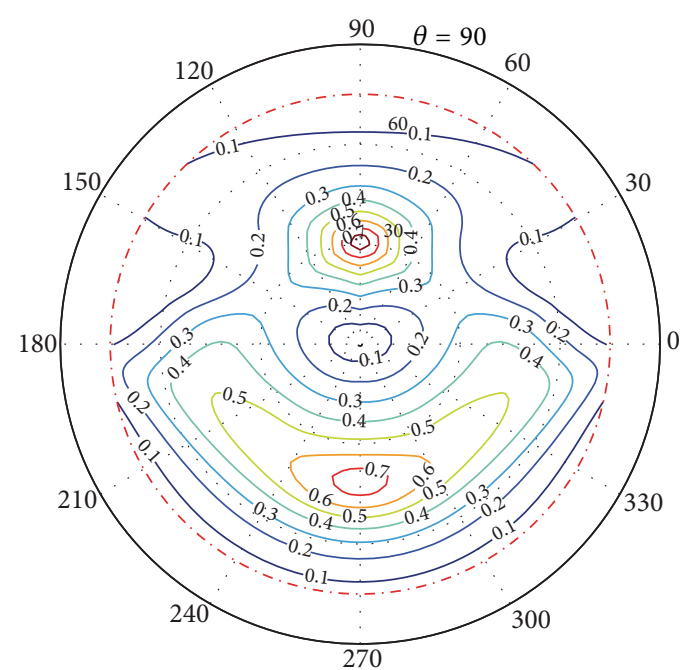

(b) $\gamma=30^{\circ}, \beta=45^{\circ}$

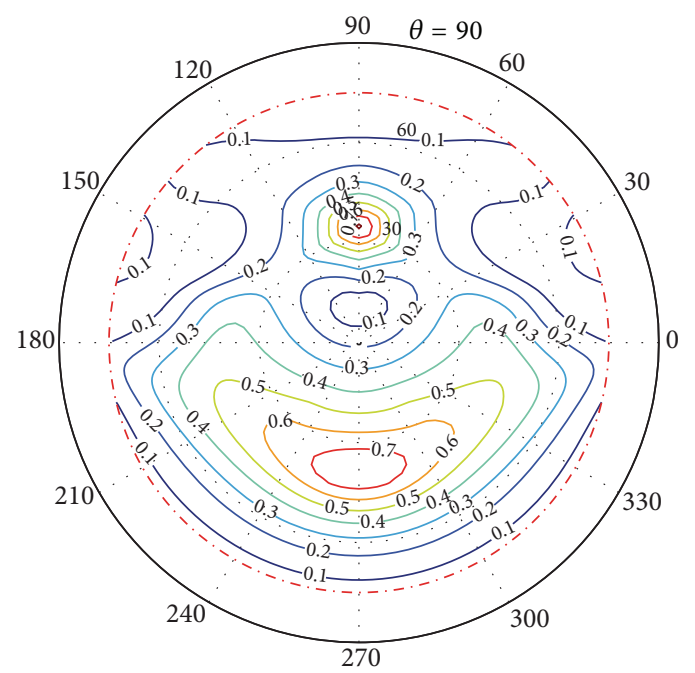

(d) $\gamma=45^{\circ}, \beta=45^{\circ}$

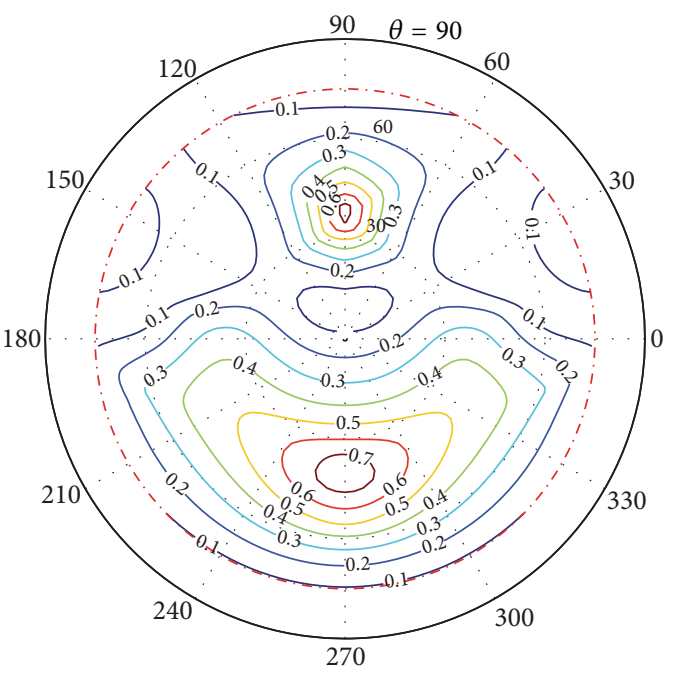

(f) $\gamma=60^{\circ}, \beta=60^{\circ}$

FIGURE 4: The dexterity distribution over the workspace with $\phi_{z}=0$. 


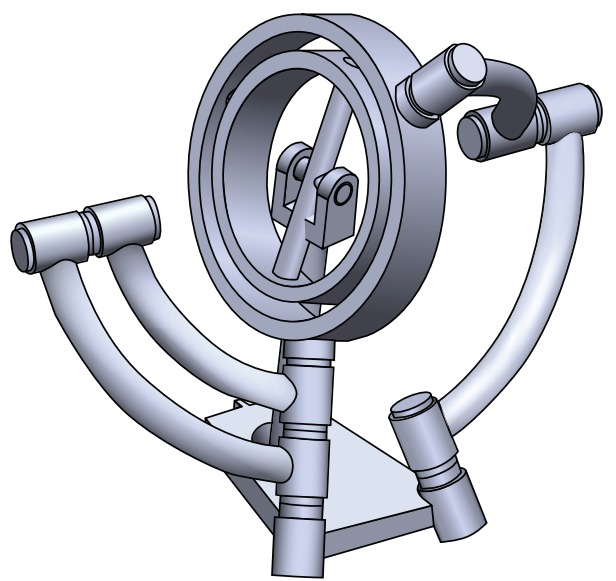

(a) $\gamma=30^{\circ}, \beta=60^{\circ} ; \phi_{x}=-4.526^{\circ}, \phi_{y}=73.221^{\circ}$

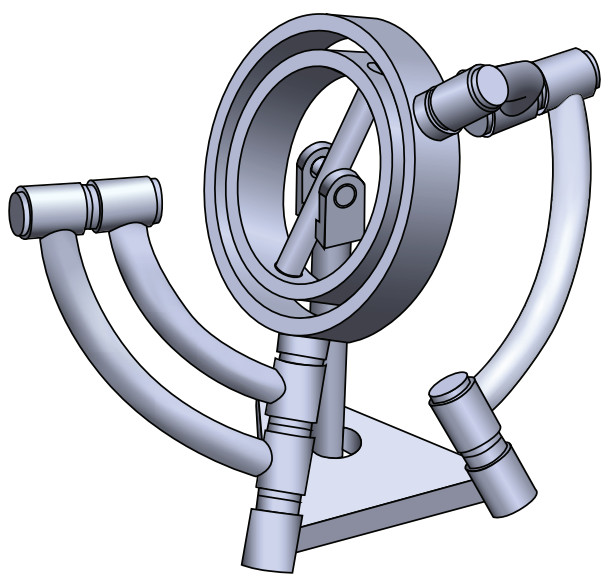

(c) $\gamma=45^{\circ}, \beta=60^{\circ} ; \phi_{x}=-10.104^{\circ}, \phi_{y}=65.905^{\circ}$

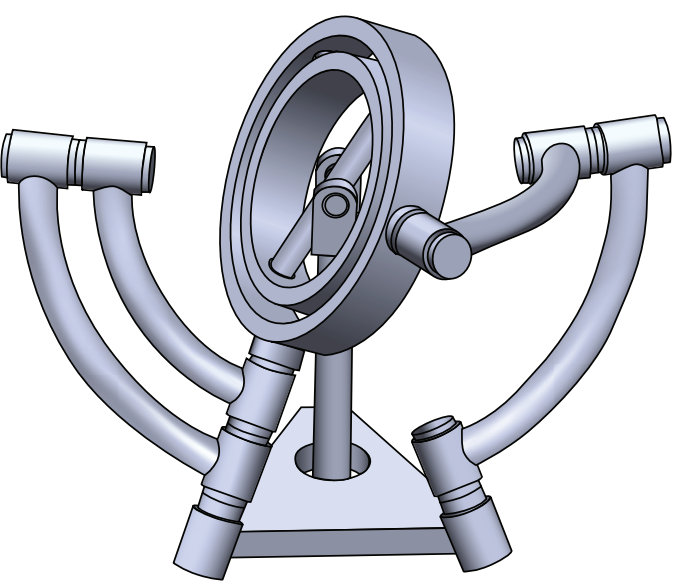

(b) $\gamma=45^{\circ}, \beta=45^{\circ} ; \phi_{x}=-26.899^{\circ}, \phi_{y}=60^{\circ}$

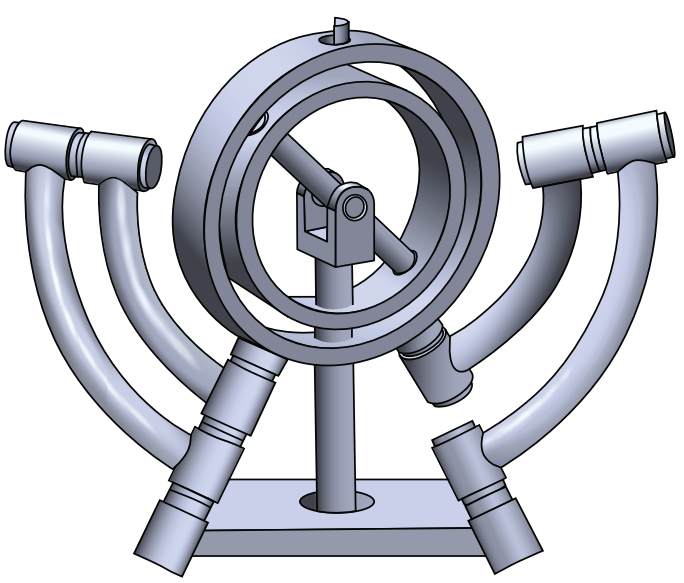

(d) $\gamma=60^{\circ}, \beta=30^{\circ} ; \phi_{x}=-90^{\circ}, \phi_{y}=-30^{\circ}$

FIGURE 5: Examples of Type I singularity of the asymmetrical SPM.

angle $\theta_{i}$ and Euler angles $\phi_{x}$ and $\phi_{y}$ can be calculated to describe the singular configuration. Taking the sets of parameters in Figure 4 as examples, the singularity of case $\alpha_{12}=\alpha_{1}+\alpha_{2}$ does not exist; for the case $\alpha_{12}=\alpha_{1}-\alpha_{2}$, it is found that the solutions of $\phi_{x}$ and $\phi_{y}$ corresponding to the $i$ th leg are definite and $\theta_{i}$ is subject to its motion range when $\gamma \in\left[15^{\circ}, 45^{\circ}\right], \beta \in\left[15^{\circ}, 60^{\circ}\right]$ or $\gamma \in\left[45^{\circ}, 60^{\circ}\right]$, and $\beta \in\left[15^{\circ}, 30^{\circ}\right]$, as shown in Figure 5.

From the forward Jacobian matrix, it is seen that the SPM is at Type II singularity when the normals to the planes of the distal links are linearly dependent, that is, the two distal links and the mobile platform being coplanar, which yields

$$
\begin{gathered}
\mathbf{v}_{i}=\left[\begin{array}{lll}
a_{i} & b_{i} & c_{i}
\end{array}\right]^{T}=\mathbf{Q v}_{i}^{*} ; \\
\mathbf{v}_{i}^{*}=\left[\begin{array}{lll}
(-1)^{i+1} \sin \left(\beta+\alpha_{2}\right) \cos \left(\beta+\alpha_{2}\right) & 0
\end{array}\right]^{T} .
\end{gathered}
$$

By the same token, in comparison to (2), the angles $\theta_{i}, \phi_{x}$, and $\phi_{y}$ can be found by solving the six overdetermined equation systems. Figure 6 illustrates two examples of Type
II singularity, where this type of singularity does not exist in the workspace for the second design.

Based on the above analysis, although the set of parameters in Figure 4(e) results in low GCI of the SPM, the regular workspace free of singularity is much larger than other designs. This means that smaller $\beta$ and larger $\gamma$ can make the manipulator have better performance of the workspace.

\section{Dynamic Modeling of the Manipulator}

In order to compute the power consumption effectively, all the mobile bodies will be taken into account for the dynamic modeling. The dynamics of the SPM under study can be solved by using the Lagrange equations [20] below:

$$
\frac{\mathrm{d}}{\mathrm{d} t}\left(\frac{\partial L}{\partial \dot{\mathbf{q}}}\right)-\frac{\partial L}{\partial \mathbf{q}}+\mathbf{C}_{q}^{T} \boldsymbol{\lambda}=\mathbf{Q}_{\mathrm{ex}},
$$

where $L \equiv T-V$ is the Lagrangian of the system, including the mobile platform and the three legs, and $\mathbf{q}=$ $\left[\theta_{1}, \theta_{2}, \theta_{3}, \phi_{x}, \phi_{y}, \phi_{z}\right]^{T}$. Moreover, $\mathbf{Q}_{\mathrm{ex}}=\left[\boldsymbol{\tau}^{T}, \mathbf{0}\right]^{T} \in \mathbb{R}^{6}$ is 


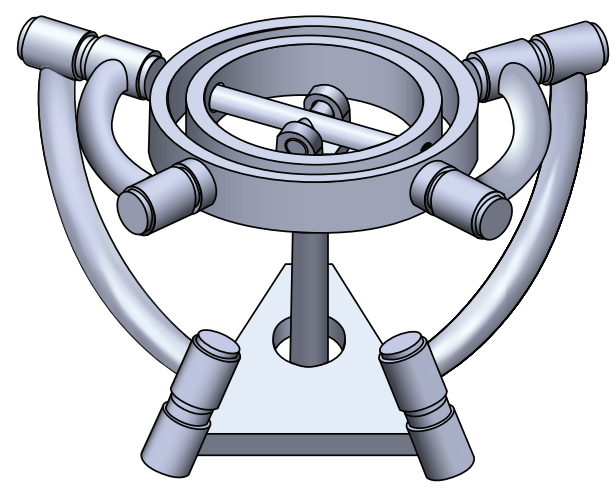

(a) $\gamma=45^{\circ}, \beta=45^{\circ} ; \phi_{x}=16.395^{\circ}, \phi_{y}=0^{\circ}$

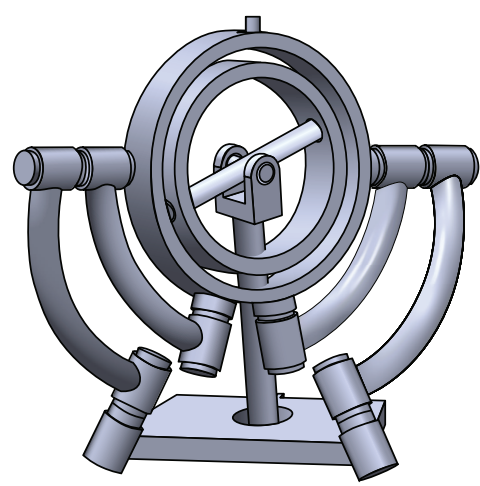

(b) $\gamma=60^{\circ}, \beta=15^{\circ} ; \phi_{x}=-73.898^{\circ}, \phi_{y}=$ $-53.293^{\circ}$

Figure 6: Examples of Type II singularity of the asymmetrical SPM.

the vector of external forces and vector $\tau=\left[\tau_{1}, \tau_{2}, \tau_{3}\right]^{T}$ characterizes the actuator torques. Matrix $\mathbf{C}_{q}$ is the system's constraint Jacobian, which can be found from the velocity equation (9), namely,

$$
\mathbf{B} \dot{\boldsymbol{\theta}}-\mathbf{A} \dot{\boldsymbol{\phi}}=\left[\begin{array}{ll}
\mathbf{B}-\mathbf{A}
\end{array}\right]\left[\begin{array}{ll}
\dot{\boldsymbol{\theta}}^{T} & \dot{\boldsymbol{\phi}}^{T}
\end{array}\right]^{T}=\mathbf{0} .
$$

Therefore, the matrix of constraints is found as $\mathbf{C}_{q}=$ [B - A ]. Moreover, $\boldsymbol{\lambda}=\left[\lambda_{1}, \lambda_{2}, \lambda_{3}\right]^{T}$ is a vector of Lagrange multipliers.

5.1. Lagrangian of the Mobile Platform. Due to the decoupled rotation between the inner and outer rings of the mobile platform, the Lagrangian of the mobile platform includes two parts as follows:

$$
L_{M}=T_{I}+T_{O}=\frac{1}{2} \boldsymbol{\omega}^{T}\left(\mathbf{R I}_{I} \mathbf{R}^{T}\right) \boldsymbol{\omega}+\frac{1}{2} \boldsymbol{\omega}^{T}\left(\mathbf{Q} \mathbf{I}_{O} \mathbf{Q}^{T}\right) \boldsymbol{\omega}^{\prime},
$$

where $\mathbf{I}_{I}$ and $\mathbf{I}_{O}$ denote the mass moment of inertia of the inner and outer rings.

5.2. Lagrangian of the Active Leg. Since all the mobile bodies of SPMs rotate about the center of rotation, from the motions of the active leg derived in Section 3.3.2, the Lagrangian of the $i$ th leg is derived as follows:

$$
\begin{aligned}
L_{i}= & T_{L i}-V_{L i} \\
= & \frac{1}{2} I_{U} \dot{\theta}_{i}^{2}+\frac{1}{2} \boldsymbol{\omega}_{i}^{T} \mathbf{I}_{L 2} \boldsymbol{\omega}_{i} \\
& -m_{l 1} \bar{x}_{1} \mathbf{g}^{T} \mathbf{h}_{i}-m_{l 2} \bar{x}_{2} \mathbf{g}^{T} \mathbf{e}_{i x}
\end{aligned}
$$

with

$$
\mathbf{I}_{L 2}=\mathbf{Q}_{i} \mathbf{I}_{L} \mathbf{Q}_{i}^{T},
$$

where $I_{U}$ is the proximal link's mass moment of inertia about $\mathbf{u}_{i}$ and $\mathbf{I}_{L}$ is the distal link's mass moment of inertia about point $O$. Moreover, $\bar{x}_{1}$ and $\bar{x}_{2}$ indicate the centers of the mass of the proximal and distal links, respectively, and $\mathbf{h}_{i}=\left(\mathbf{u}_{i}+\right.$ $\left.\mathbf{v}_{i}\right) /\left\|\mathbf{u}_{i}+\mathbf{v}_{i}\right\|$ and $\mathbf{g}=\left[0,0,9.81 \mathrm{~m} / \mathrm{s}^{2}\right]^{T}$.

Let $I_{C}$ be the moment of inertia of the center shaft, and its Lagrangian is expressed as

$$
L_{C}=\frac{1}{2} I_{C} \dot{\theta}_{3}^{2} .
$$

5.3. Lagrangian of the Passive Leg. The Lagrangian of the passive leg is derived as

$$
\begin{aligned}
L_{P}= & T_{P}-V_{P} \\
= & \frac{1}{2} I_{l 4} \dot{\delta}^{2}+\frac{1}{2} I_{l 3} \omega_{l 3}^{2}+\frac{1}{2} m_{l 3} v_{l 3}^{2} \\
& -m_{l 4} g\left[\frac{1}{2} l_{4} \sin \left(\delta-\phi_{0}\right)-h\right] \\
& -\frac{1}{2} m_{l 3} g\left[l_{1} \sin \phi_{x}+l_{4} \sin \left(\delta-\phi_{0}\right)-h\right],
\end{aligned}
$$

where $m_{l 3}, I_{l 3}$ and $m_{l 4}, I_{l 4}$ are the mass and moment of inertia for link $l_{3}$ and $l_{4}$, respectively.

Substituting the previous Lagrangian into (29), the terms in the equation of motion for this dynamic system [20] can be derived. With an external moment vector $\mathbf{m}$, the actuator torques are expressed as

$$
\boldsymbol{\tau}_{a}=\boldsymbol{\tau}-\mathbf{J}^{T} \mathbf{m} .
$$

\section{Numerical Simulation}

In accordance with Section 4, dynamic characteristics of the manipulator with parameters of Figure 4(d) will be presented 

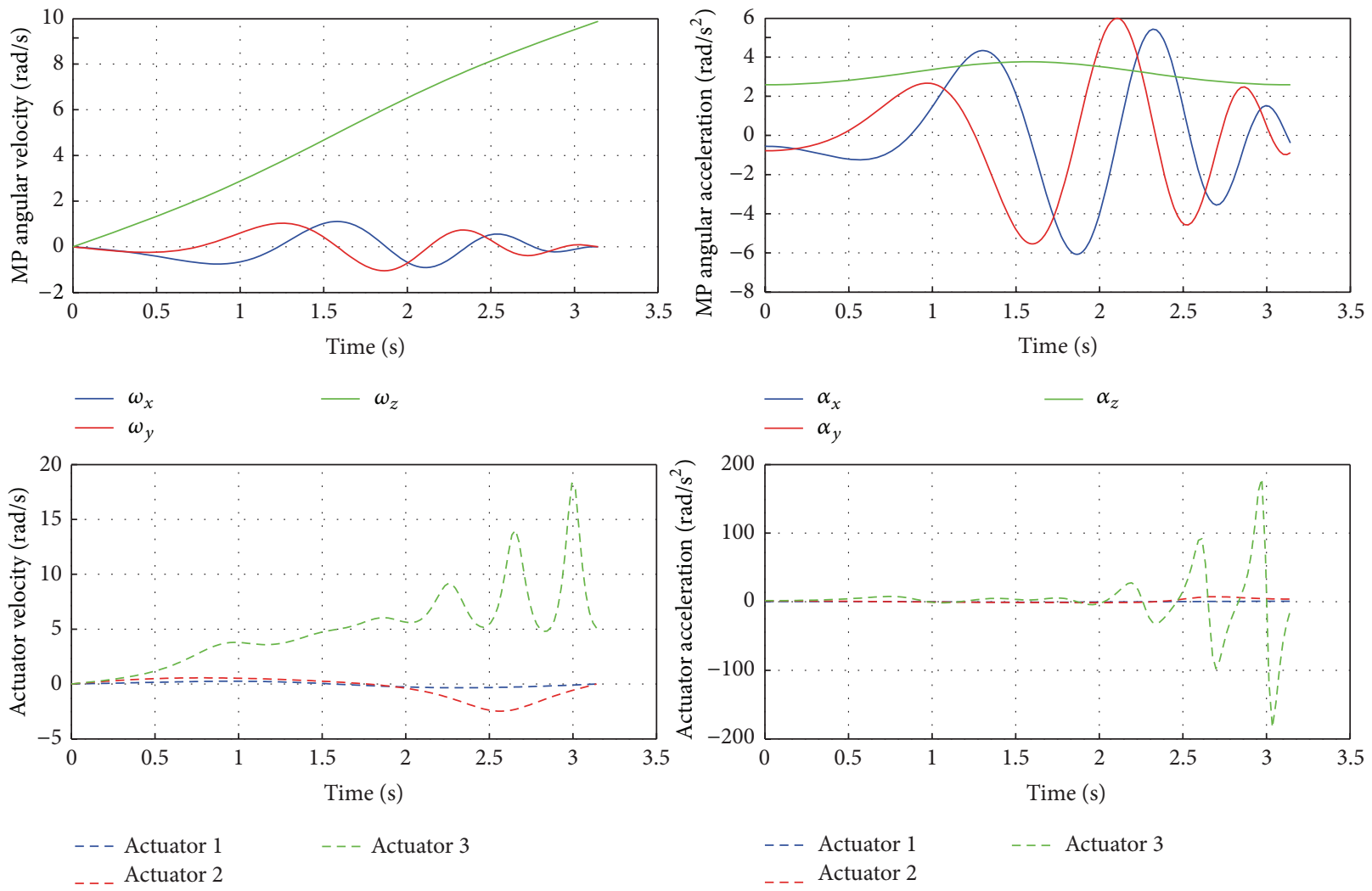

(a)
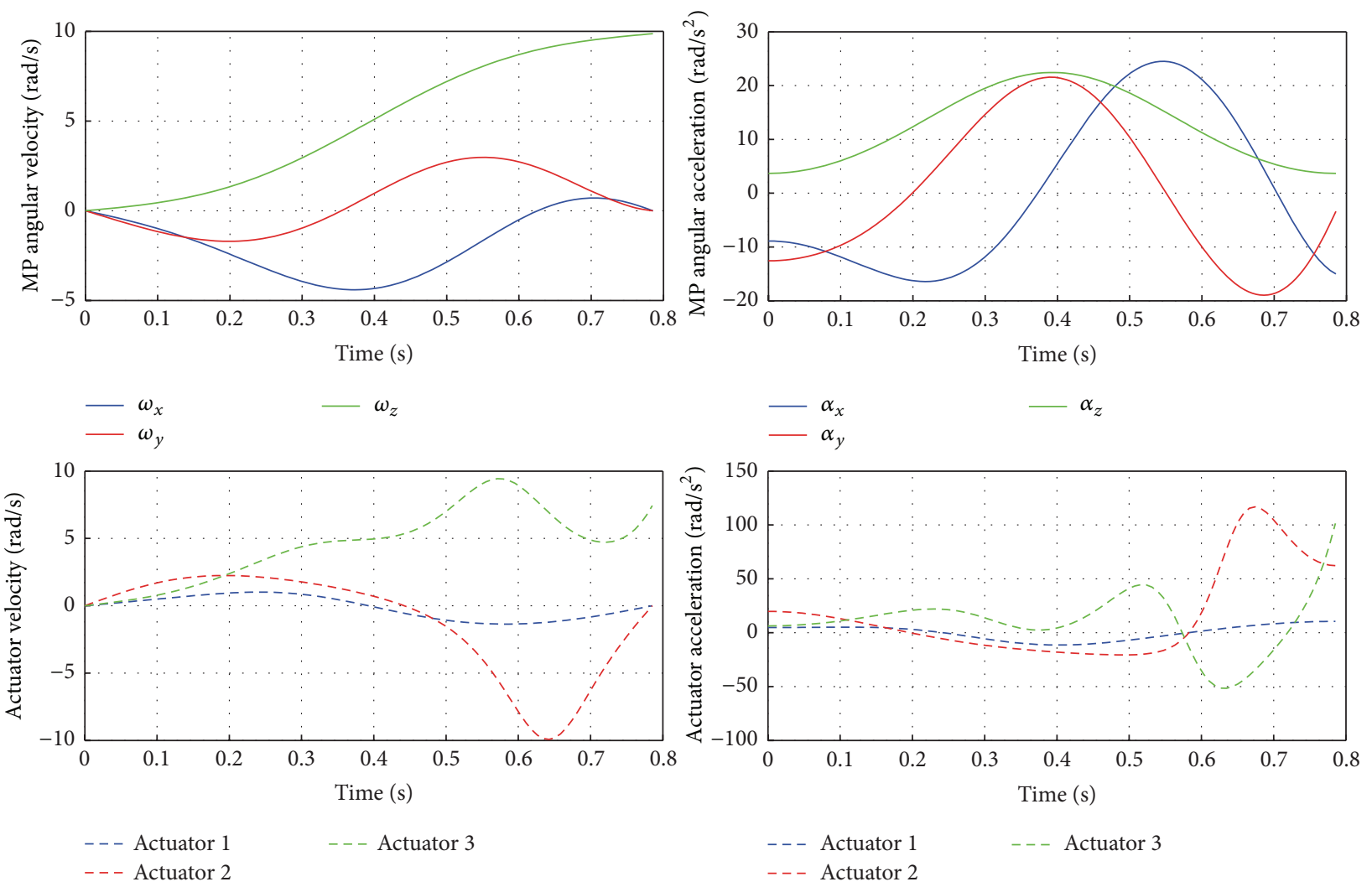

(b)

FIGURE 7: The velocity and acceleration profiles for the simulations: (a) trajectory 1; (b) trajectory 2. 

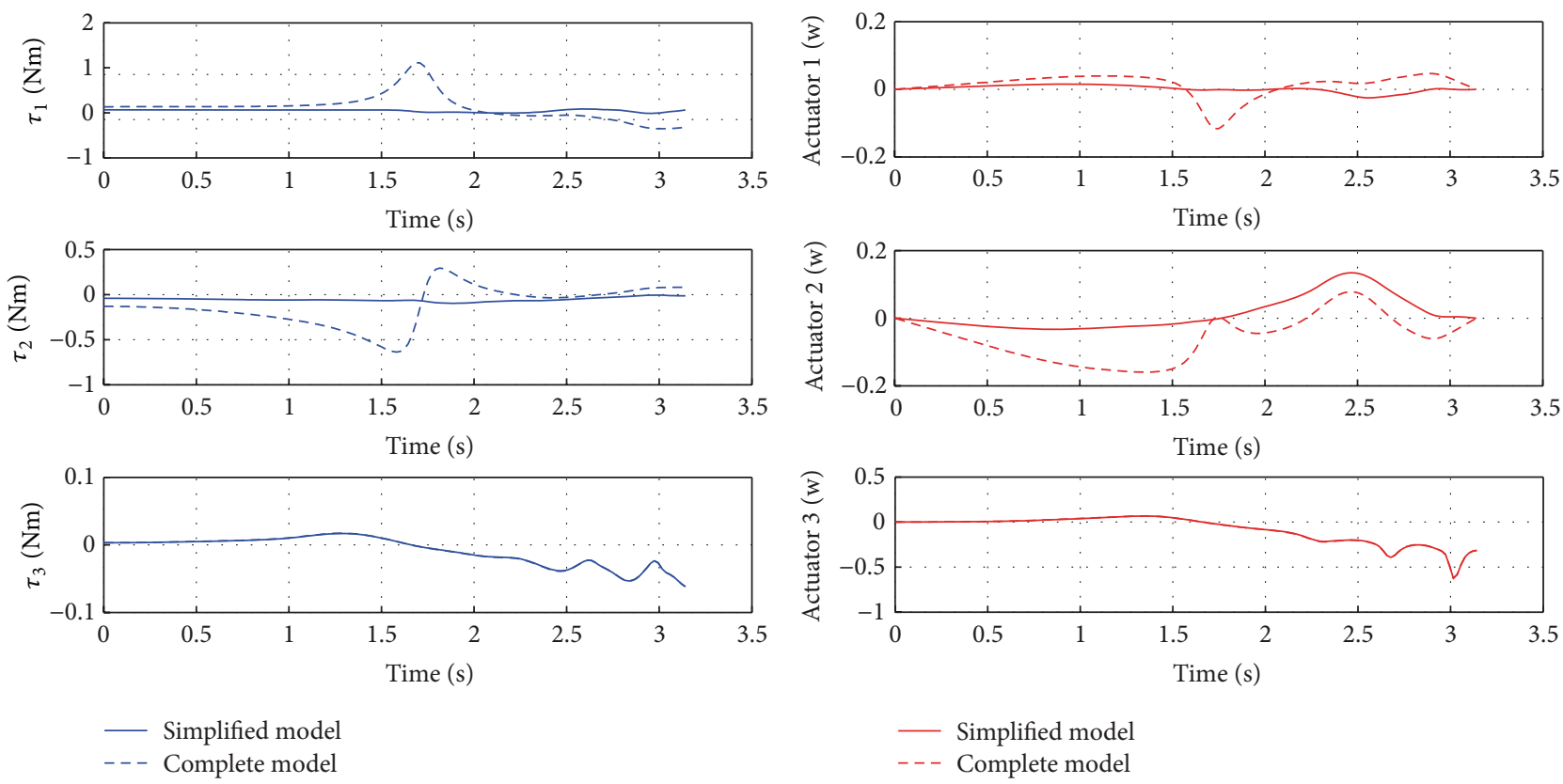

(a)

(b)

FIGURE 8: The dynamic simulation results of trajectory 1: (a) actuator torque; (b) actuator power.

through simulation, where the dimensions and mass properties are listed below:

$$
\begin{gathered}
\mathbf{I}_{I}=\operatorname{diag}\left[\begin{array}{lll}
0.4821 & 0.4668 & 0.9133
\end{array}\right] \cdot 10^{-3} \mathrm{~kg} \mathrm{~m}^{2}, \\
\mathbf{I}_{O}=\operatorname{diag}\left[\begin{array}{lll}
1.2518 & 1.2389 & 0
\end{array}\right] \cdot 10^{-3} \mathrm{~kg} \mathrm{~m}^{2}, \\
m_{l 1}=0.246 \mathrm{~kg}, \\
I_{U}=8.1062 \cdot 10^{-4} \mathrm{~kg} \mathrm{\textrm {m } ^ { 2 } ,} \quad \bar{x}_{1}=0.084 \mathrm{~m}, \\
I_{C}=4.4226 \cdot 10^{-5} \mathrm{~kg} \mathrm{\textrm {m } ^ { 2 }} \\
m_{l 2}=0.132 \mathrm{~kg}, \\
\mathbf{I}_{L}=\left[\begin{array}{ll}
0.6452 \quad & 0.0973 \quad 0.7425
\end{array}\right] \cdot 10^{-3} \mathrm{~kg} \mathrm{~m}{ }^{2} \\
\bar{x}_{2}=0.069 \mathrm{~m}, \\
m_{l 3}=0.050 \mathrm{~kg}, \quad I_{l 3}=8.1667 \cdot 10^{-5} \mathrm{~kg} \mathrm{~m}^{2} \\
m_{l 4}=0.044 \mathrm{~kg}, \quad I_{l 4}=1.2959 \cdot 10^{-4} \mathrm{~kg} \mathrm{~m}^{2} \\
h=0.103 \mathrm{~m}, \quad d=0.041 \mathrm{~m}, \quad l_{2}=0.088 \mathrm{~m}, \\
l 3=0.104 \mathrm{~m}, \quad l 4=0.080 \mathrm{~m}^{2}
\end{gathered}
$$

Based on the kinematic and dynamic models, the inverse dynamic simulation is conducted with low/high-speed motions defined by the following two trajectories:

$$
\begin{array}{r}
\phi_{x}=\frac{\pi}{4} \cos (t), \quad \phi_{y}=\frac{\pi}{4} \cos (t), \quad \phi_{z}=\frac{\pi}{2} t^{2} \\
t \in[0, \pi] \mathrm{s}
\end{array}
$$

(2) $\phi_{x}=\frac{\pi}{4} \cos (4 t)$

$$
\begin{array}{r}
\phi_{y}=\frac{\pi}{4} \cos (4 t), \quad \phi_{z}=2 \pi t^{2}, \\
t \in\left[0, \frac{\pi}{4}\right] \mathrm{s},
\end{array}
$$

where the velocities and accelerations of the mobile platform and the actuators for the two trajectories are displayed in Figures $7(\mathrm{a})$ and $7(\mathrm{~b})$, respectively. Through comparison, it is found that the difference of the input velocities will reduce when the manipulator is at high-speed motion. Hence, the input requirements on the actuators will be more isotropic.

The corresponding simulation results, with the comparison of a simplified model where passive and distal links are not considered, are illustrated with Figures 8 and 9. It is seen that the gravity and inertial forces of the passive and distal links strongly influence the output torques and powers of the two parallel actuators, especially in the region with poor dexterity. At some orientations, the magnitudes of the actuator torque/power of the completed model are smaller than the simplified one, and the reason is that the torque applied to the actuator caused by the links' gravity has the same direction with the accelerations. On the other hand, the gravity and inertial forces have less influence on the third actuator due to the decoupled mobility.

Figure 10 demonstrates the actuator torques/powers when the manipulator tracks the second trajectory with an external moment $\mathbf{m}=[1,1,1] \mathrm{Nm}$ in the Cartesian space exerted on the mobile platform. It says that the torques/powers profiles are quite close; hence, the simplified model can be used to approximate the manipulator's dynamic characteristics when the external moment has much more influence than the inertial forces. All the three cases result in 

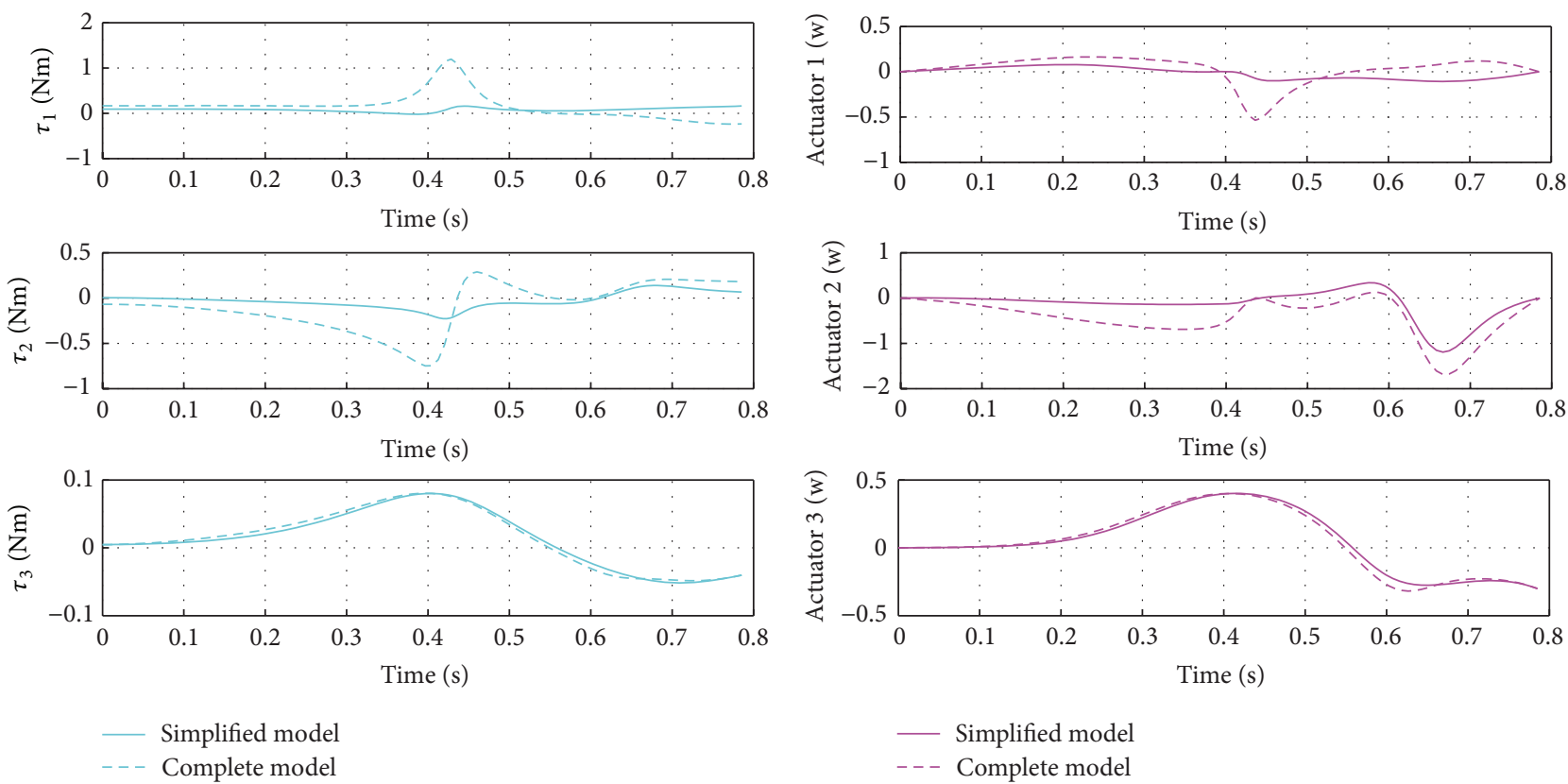

(a)

(b)

FIGURE 9: The dynamic simulation results of trajectory 2: (a) actuator torque; (b) actuator power.
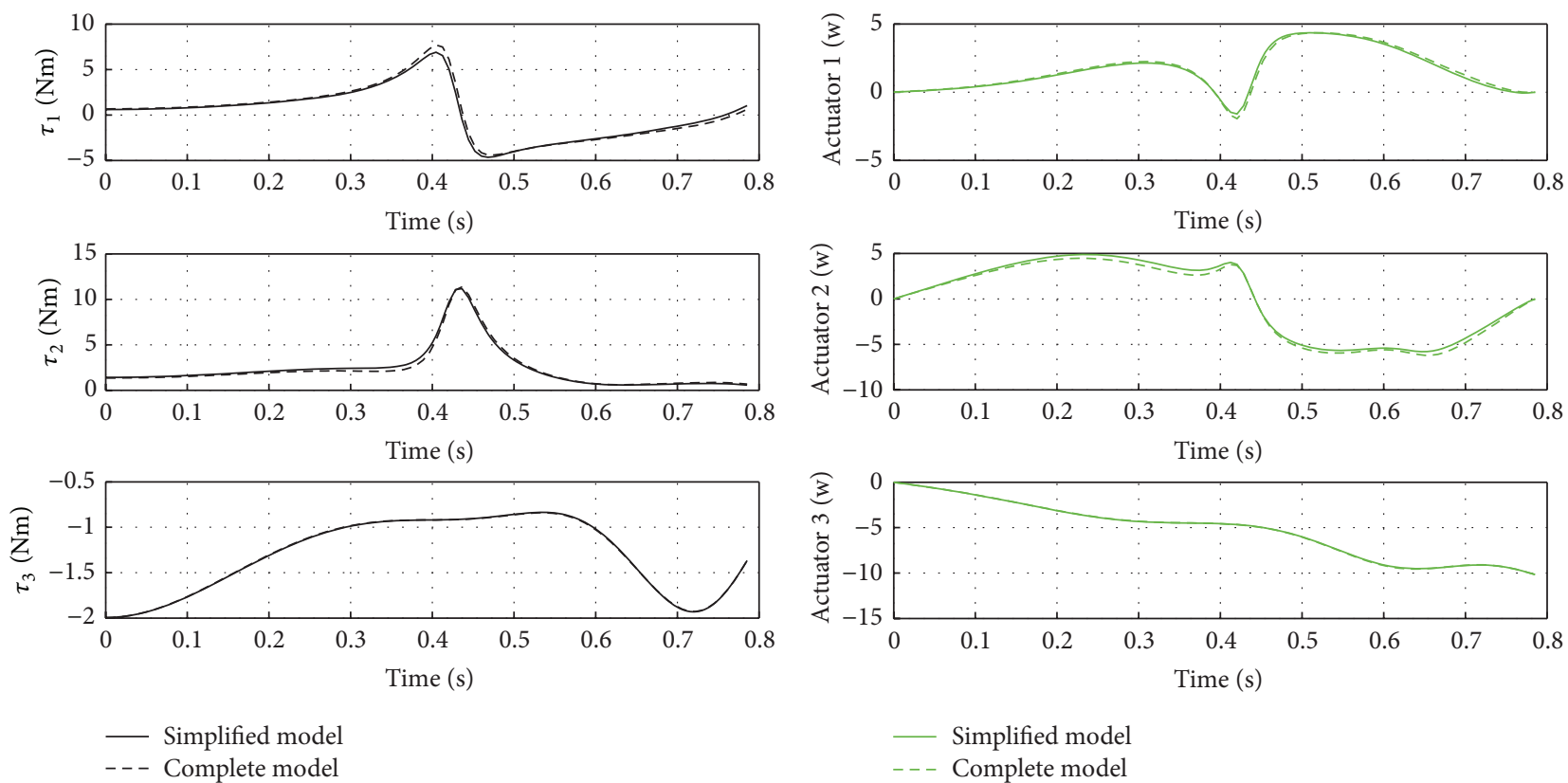

(a)

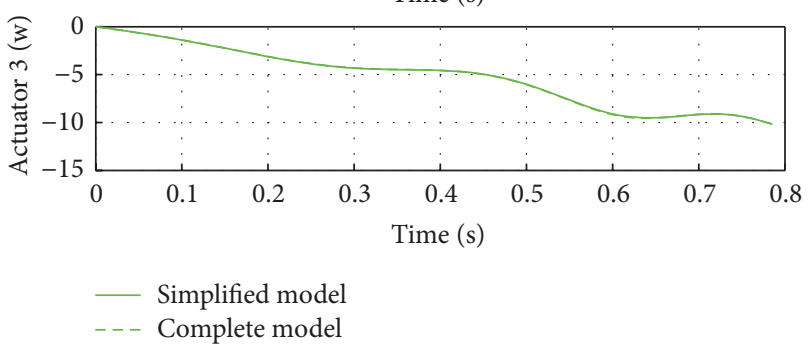

(b)

FIGURE 10: The dynamic simulation results of trajectory 2 with external moment: (a) actuator torque; (b) actuator power.

the smallest torque requirement on the third actuator but its output power is the highest among the three actuators.

\section{Conclusion}

This paper introduces an asymmetrical parallel robotic wrist which admits a large orientation workspace. Its unique structure allows that the manipulator can generate a decoupled unlimited-torsion rotation and ensures high positioning accuracy. This proposed manipulator can be used as a tool head in the complicated surface machining, such as milling, and also can work as an active spherical joint.

The kinematic performance of the proposed manipulator is studied and performance contours of the dexterity over 
the workspace are visualized for the optimum design in the further study. Moreover, the singularity is analyzed by solving the geometric constraint equations. Using the classical method of Lagrange multipliers, the equations of motion for the manipulator under study were derived. All the moving bodies are taken into account to describe this dynamic system effectively and clearly. Numerical simulations show that some simplified models that neglect the weight of intermediate bodies cannot characterize the dynamics of the system appropriately. The developed dynamic model can be used for the actuation selection or dynamics evaluation. In the future, the optimal design of the manipulator will be conducted for improvement of dexterity and reduction of energy consumption.

\section{Nomenclature}

L: $\quad$ The Lagrangian of the robotic system

$\boldsymbol{\omega}, \boldsymbol{\omega}^{\prime}, \boldsymbol{\omega}_{i}$ : The angular velocities of the inner/outer rings and the $i$ th distal link

$\tau_{a}: \quad$ Vector of the actuator torque

$\delta$ : $\quad$ Output angle of the four-bar linkage of the passive leg

$\dot{\phi}: \quad$ The angle rates of the inner ring

A, B: $\quad$ Forward and backward kinematic Jacobians of the inner ring

$\mathrm{C}_{q}$ : $\quad$ Constraint Jacobian matrix

J: Kinematic Jacobian matrix

$\mathbf{J}_{x}, \mathbf{J}_{q}: \quad$ Forward and backward kinematic Jacobians of the outer ring

Q, R: Rotation matrix of the outer and inner rings of the mobile platform

$\mathbf{Q}_{i}$ : $\quad$ Transformation matrix of the $i$ th distal link

$\mathbf{u}_{i}$ : $\quad$ Unit vector of the input axis in the $i$ th leg

$\mathbf{v}_{i}$ : Unit vector of the intermediate revolute joint in the $i$ th leg

$\mathbf{w}_{i}$ : Unit vector of the top revolute joint in the $i$ th leg

$\theta_{i}: \quad$ The $i$ th input angle.

\section{Conflict of Interests}

The author declares that there is no conflict of interests regarding the publication of this paper.

\section{References}

[1] C. M. Gosselin and J. F. Hamel, "The Agile Eye: a high-performance three-degree-of-freedom camera-orienting device," in Proceedings of the IEEE International Conference on Robotics and Automation, vol. 1, pp. 781-786, San Diego, Calif, USA, May 1994.

[2] T. Li and S. Payandeh, "Design of spherical parallel mechanisms for application to laparoscopic surgery," Robotica, vol. 20, no. 2, pp. 133-138, 2002.

[3] H. Asada and J. Granito, "Kinematic and static characterization of wrist joints and their optimal design," in Proceedings of the
IEEE International Conference on Robotics and Automation, pp. 244-250, 1985

[4] G. Wu, S. Bai, and J. Kepler, "Mobile platform center shift in spherical parallel manipulators with flexible limbs," Mechanism and Machine Theory, vol. 75, pp. 12-26, 2014.

[5] C. M. Gosselin and J. Angeles, "The optimum kinematic design of a spherical three-degree-of-freedom parallel manipulator," Journal of Mechanical Design, vol. 111, no. 2, pp. 202-207, 1989.

[6] S. Bai, "Optimum design of spherical parallel manipulators for a prescribed workspace," Mechanism and Machine Theory, vol. 45 , no. 2, pp. 200-211, 2010.

[7] C. Gosselin and J. Angeles, "Global performance index for the kinematic optimization of robotic manipulators," Journal of Mechanical Design, vol. 113, no. 3, pp. 220-226, 1991.

[8] S. Bai, M. R. Hansen, and T. O. Andersen, "Modelling of a special class of spherical parallel manipulators with Euler parameters," Robotica, vol. 27, no. 2, pp. 161-170, 2009.

[9] G. Wu, "Multiobjective optimum design of a 3-RRR spherical parallel manipulator with kinematic and dynamic dexterities," Modeling, Identification and Control, vol. 33, no. 3, pp. 111-121, 2012.

[10] I. A. Bonev and C. M. Gosselin, "Singularity loci of spherical parallel mechanisms," in Proceedings of the IEEE International Conference on Robotics and Automation, pp. 2957-2962, April 2005.

[11] F. Bidault, C. P. Teng, and J. Angeles, "Structural optimization of a spherical parallel manipulator using a two-level approach," in Proceedings of the ASME Design Engineering Technical Conference and Computers and Information in Engineering Conference, pp. 215-224, Pittsburgh, Pa, USA, September 2001.

[12] X. Kong and C. M. Gosselin, "Type synthesis of three-degreeof-freedom spherical parallel manipulators," The International Journal of Robotics Research, vol. 23, no. 3, pp. 237-245, 2004.

[13] G. Gogu, "Fully-isotropic three-degree-of-freedom parallel wrists," in Proceedings of the IEEE International Conference on Robotics and Automation (ICRA '07), pp. 895-900, Roma, Italy, April 2007.

[14] M. Karouia and J. M. Hervé, "Asymmetrical 3-dof spherical parallel mechanisms," European Journal of Mechanics: A/Solids, vol. 24, no. 1, pp. 47-57, 2005.

[15] S. Caro, D. Chablat, R. Ur-Rehman, and P. Wenger, "Multiobjective design optimization of 3-PRR planar parallel manipulators," in Global Product Development, A. Bernard, Ed., pp. 373-383, Springer, Berlin, Germany, 2011.

[16] R. N. Murray, Z. X. Li, and S. S. Sastry, A Mathematical Introduction to Robotic Manipulation, CRC Press, 1994.

[17] R. Featherstone and D. Orin, "Robot dynamics: equations and algorithms," in Proceedings of the IEEE International Conference on Robotics and Automation (ICRA '00), pp. 826-834, April 2000.

[18] J. Angeles, Fundamentals of Robotic Mechanical Systems: Theory, Methods, and Algorithms, Springer, New York, NY, USA, 2007.

[19] S. Staicu, "Recursive modelling in dynamics of Agile Wrist spherical parallel robot," Robotics and Computer-Integrated Manufacturing, vol. 25, no. 2, pp. 409-416, 2009.

[20] J. G. de Jalon and E. Bayo, Kinematic and Dynamic Simulation of Multibody Systems: The Real-Time Challenge, Springer, New York, NY, USA, 1994.

[21] http://en.wikipedia.org/wiki/Universal_joint.

[22] F. Freudenstein, "Approximate synthesis of four-bar linkages," Resonance, vol. 15, no. 8, pp. 740-767, 2010. 
[23] R. E. Stamper, L.-W. Tsai, and G. C. Walsh, "Optimization of a three DOF translational platform for well-conditioned workspace," in Proceedings of the IEEE International Conference on Robotics and Automation, vol. 4, pp. 3250-3255, Albuquerque, NM, USA, 1997.

[24] X.-J. Liu, J. Wang, K.-K. Oh, and J. Kim, "A new approach to the design of a DELTA robot with a desired workspace," Journal of Intelligent and Robotic Systems, vol. 39, no. 2, pp. 209-225, 2004.

[25] P. Wenger and D. Chablat, "Kinematic analysis of a new parallel machine tool: the Orthoglide," in Advances in Robot Kinematics, J. Lenarčič and M. M. Stanišić, Eds., pp. 305-314, Springer, Amsterdam, The Netherlands, 2000. 

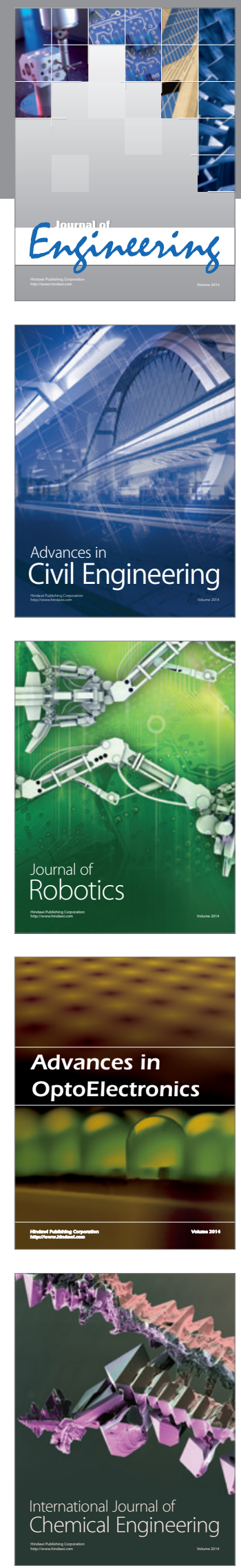

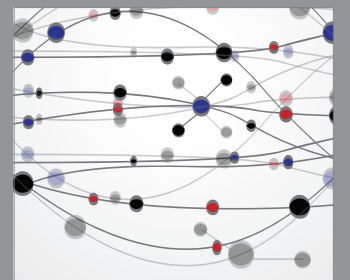

The Scientific World Journal
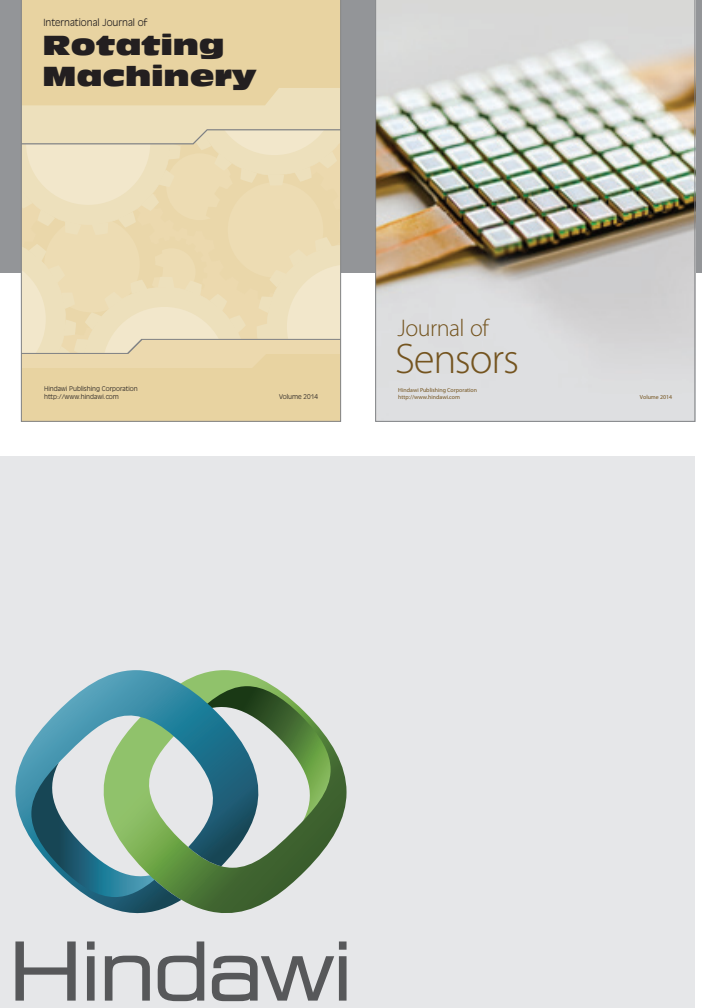

Submit your manuscripts at http://www.hindawi.com
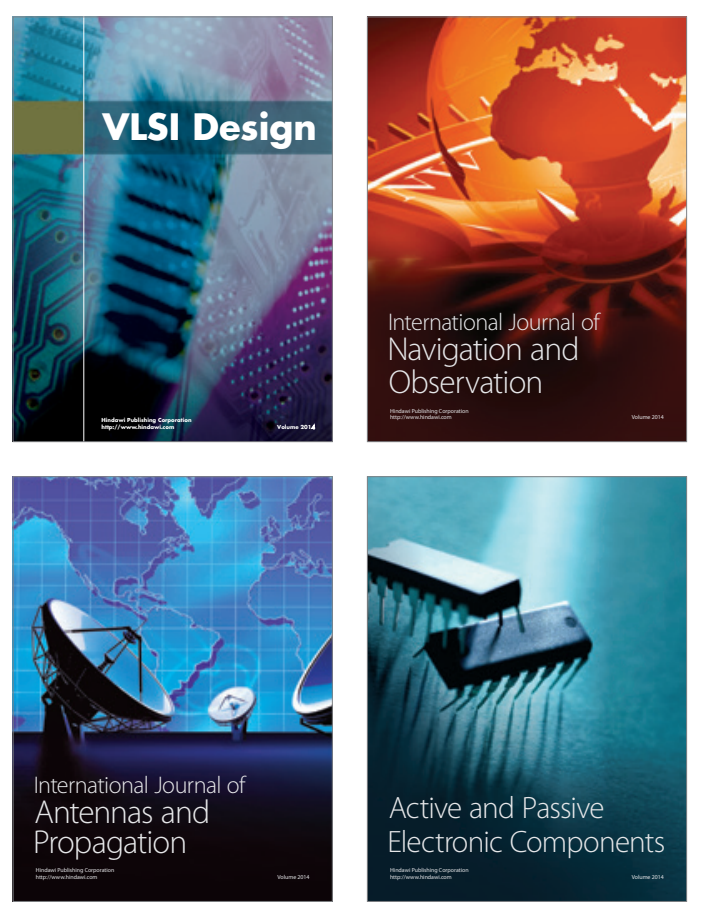
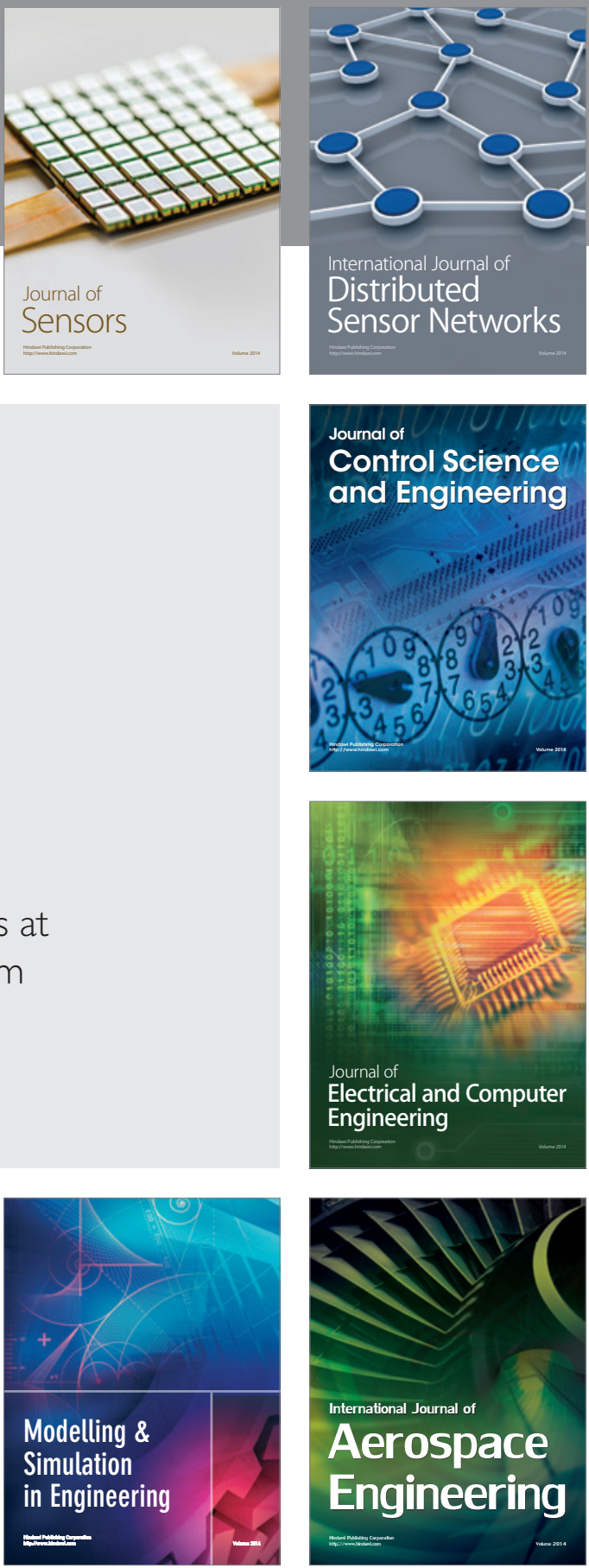

Journal of

Control Science

and Engineering
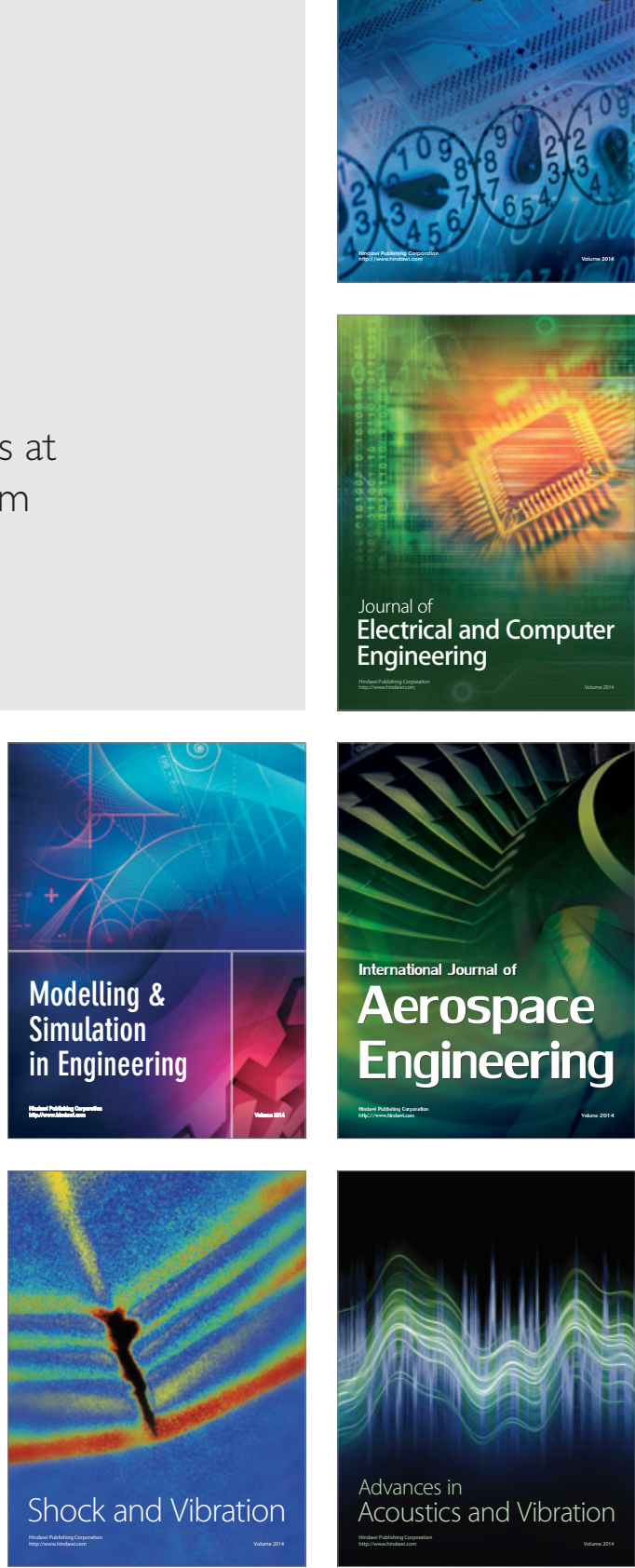